\title{
Anodic stripping voltammetric analysis of different species of copper in seawater using an electrochemical sensor
}

\author{
Xueping $\mathrm{Hu}^{1,2} \cdot$ Dawei $\operatorname{Pan}^{1,2} \oplus \cdot$ Haitao Han ${ }^{1,2} \cdot$ Chenchen Wang ${ }^{1}$
}

Received: 21 January 2018 / Revised: 29 June 2018 / Accepted: 15 July 2018 / Published online: 30 July 2018

(c) The Oceanographic Society of Japan and Springer Japan KK, part of Springer Nature 2018

\begin{abstract}
The behavior, effects, and toxicity of an element often depend largely on its speciation. In this study, the speciation of copper $(\mathrm{Cu})$ in seawater was investigated via the fixed-point determination of electroactive $\mathrm{Cu}$, acid-dissolved $\mathrm{Cu}$, inert $\mathrm{Cu}$, and total dissolved $\mathrm{Cu}$ using a voltammetric method with an electrochemical sensor. This method was found to be simple and effective for speciation analysis as no complicated pretreatment or separation steps were required, permitting its use for the direct determination of $\mathrm{Cu}$ speciation in real water samples with confirmed accuracy. Moreover, following simple electrolytic cleaning, the sensor exhibited good measurement stability and reproducibility when it was reused. Fixed-point determinations of electroactive $\mathrm{Cu}$, acid-dissolved $\mathrm{Cu}$, inert $\mathrm{Cu}$, and total dissolved $\mathrm{Cu}$ in local seawater were carried out for 15 days. The total dissolved $\mathrm{Cu}$ concentration ranged from 45.03 to $122.85 \mathrm{nM}$, and its average concentration was $66.05 \mathrm{nM}$. The speciation of $\mathrm{Cu}$ was influenced by external environmental changes to some extent but was observed to remain relatively constant. The correlations between the concentrations of different $\mathrm{Cu}$ species and environmental parameters are discussed here in detail.
\end{abstract}

Keywords Voltammetric determination $\cdot \mathrm{Cu} \cdot$ Speciation $\cdot$ Seawater $\cdot$ Environmental parameters

\section{Introduction}

The rapid increases in environmental metal pollution levels observed in recent decades have resulted in heightened concern over both public and environmental health. Traditionally, analytical measurements performed for environmental monitoring focused on the total content of each element of interest (e.g., toxic elements such as lead, mercury, and cadmium, as well as elements utilized in living organisms such as cobalt, selenium, and magnesium) in a sample (Kot and Namiesnik 2000). But, as knowledge advanced, it was soon realized that data on total elemental concentrations were

Electronic supplementary material The online version of this article (https://doi.org/10.1007/s10872-018-0491-z) contains supplementary material, which is available to authorized users.

Dawei Pan

dwpan@yic.ac.cn

1 Key Laboratory of Coastal Environmental Processes and Ecological Remediation, Yantai Institute of Coastal Zone Research, Chinese Academy of Sciences, Yantai 264003, People's Republic of China

2 University of Chinese Academy of Sciences, Beijing 100049, People's Republic of China insufficient. Biochemical and toxicological investigations have shown that the behavior and effects of a trace metal greatly depend upon its speciation (Adnívia et al. 2016; Florence 1986; Huang and Wang 2003). Therefore, it is essential to determine the relative abundances of the species of each trace element in varying environments.

Copper $(\mathrm{Cu})$ is an important trace element that is required for the proper functioning of biological processes within aquatic organisms (Grosell and Wood 2002; de Polo and Scrimshaw 2012; Jacquot et al. 2014). However, $\mathrm{Cu}$ is toxic at increased concentrations as it interferes with ion transport (Tait et al. 2016; Whitby and van den Berg 2015). The toxicity of $\mathrm{Cu}$ is also dependent on its speciation (Tait et al. 2016; Guo et al. 2010). Cu is mostly found in inorganic and organic complexes in the environment, and this complexation decreases the fraction of $\mathrm{Cu}$ available to interact with organisms and to cause toxicity (Santore et al. 2012). The concentration of free $\mathrm{Cu}^{2+}$ ranges from 0.1 to $2 \mathrm{nM}$, which is toxic to a variety of ecologically important phytoplankton and zooplankton species (Byrne and Miller 1985). Research by Batley and Florence (1971) established that there are seven potential chemical and physical forms of metal ions in aquatic environments, such as free metal ions, labile metals weakly complexed with organic compounds, metals strongly 
bound in organic complexes; and so on. Among others, van den Berg proposed the concept of active ions and established a method for detecting them using electrochemical methods (Gledhill and van den Berg 1995). The concentration of labile $\mathrm{Cu}$ was determined by Huang and Wang using differential pulse anodic stripping voltammetry (Huang and Wang 2003). The correlation between the ASV-labile concentration of $\mathrm{Cu}$ and the toxicity derived from bioassays can be useful for predicting the toxicity of $\mathrm{Cu}$.

The conditional stability constant reflects the strength of an interaction between a metal ion and a ligand. The conditional stability constant of an organic $\mathrm{Cu}^{2+}$ complex, $K$, is defined as $K=[\mathrm{CuL}] /\left[\mathrm{Cu}^{2+}\right]\left[\mathrm{L}^{\prime}\right]$, where $[\mathrm{CuL}]$ is the concentration of $\mathrm{Cu}$ complexed by natural ligands, $\left[\mathrm{Cu}^{2+}\right]$ is the $\mathrm{Cu}$ ion concentration, and $\left[\mathrm{L}^{\prime}\right]$ is the concentration of the free ligand. Hirose divided the organic ligands in seawater into three classes: very strong $(\log K>13)$, strong $(\log K \approx 12)$, and weak $(\log K<10)$ (Hirose 1994). In an attempt to further simplify speciation analysis processes, we decided to divide the total pool of metal speciation present in water samples into four speciation groups: electroactive metals, aciddissolved metals, inert metals, and total dissolved metals. Electroactive metals were defined as ions that are directly detectable by electrochemical methods without any additional processing. While they are generally present in low concentrations, they are associated with high bioavailability and toxicity in marine environments. Metal ions have been found to complex rapidly with any organic matter present (Hirose 2006; Abdelraheem et al. 2017), and acid-dissolved metals were defined as metal ions that form complexes that are easily destroyed by acidic environments. Acid-dissolved metals have been found to possess relatively low activity but high levels of bioavailability and absorption by surrounding ecosystems (Guo et al. 2010; Huang and Wang 2003; Tait et al. 2016; Hirose 1994). Inert metals were defined as metal ions that strongly couple with an organic complex, leading to high levels of stability and low bioavailability. In terms of quantification, the concentration of the inert metal was considered to be equivalent to the total metal concentration minus the acid-dissolved metal and electroactive metal fractions. A significant benefit of the method proposed here is that water samples can be divided up and then easily prepared for electroanalysis using either filtration, acidification, or digestion, allowing different metal species to be readily identified and detected.

In the study reported in the present paper, after carrying out simple preparation processes, different $\mathrm{Cu}$ species in seawater samples were detected and quantified using a sensitive electrochemical detector. As well as being an efficient, straightforward, and inexpensive technique for speciation analysis, this technique was observed to be highly stable and reproducible when employed for the long-term determination of $\mathrm{Cu}$ species in marine environments.

\section{Experimental}

\subsection{Instruments}

An electrochemical workstation (CHI 660D, Chenhua Instruments, Shanghai, China) was used for all electrochemical experiments. The electrochemical sensor contained a conventional three-electrode system consisting of a Au-film-modified glassy carbon working electrode ( $\mathrm{Au} / \mathrm{GCE}, 3 \mathrm{~mm}$ in diameter), a platinum foil auxiliary electrode, and a silver chloride reference electrode $(\mathrm{Ag} /$ $\mathrm{AgCl}, 3 \mathrm{M} \mathrm{KCl}$ ). All potentials were measured against the $\mathrm{Ag} / \mathrm{AgCl}$ reference electrode. The electrochemical UV digestion apparatus employed in this work was supplied by the Marine Electrochemistry Group (Department of Earth and Ocean Sciences, School of Environmental Science, University of Liverpool, UK). UV light was generated by a high-pressure mercury vapor lamp with a power output of $125 \mathrm{~W}$, which peaked at around $250 \mathrm{~nm}$. The sample collector (Taiheng Plastics Corporation, Henan, China) was filled with $0.1 \mathrm{M} \mathrm{HCl}$ (analytical grade) for at least one day and then thoroughly washed with deionized water before use. A portable $\mathrm{pH} /$ temperature measurement apparatus (pH-100B, Bangxi Scientific Instruments Corporation, Shanghai, China) was used for in situ measurements of water characteristics and parameters during sampling. Inductively coupled plasma mass spectrometry (ICP-MS, ELAN DRC, PerkinElmer, Waltham, MA, USA) was carried out to quantitatively analyze $\mathrm{Cu}$ species, and the resulting dataset was compared with the datasets obtained using other methods.

\subsection{Chemicals and materials}

The stock standard solution of $1000 \mathrm{mg} \mathrm{L}^{-1} \mathrm{Cu}$ was supplied by the National Research Center for Certified Reference Materials (CRMs; Beijing, China). Separate standard solutions were prepared by diluting the stock solution with $10 \% \mathrm{v} / \mathrm{v} \mathrm{HCl} . \mathrm{HAuCl}_{4}$ stock liquid was purchased from Sinopharm Chemical Reagent Co. (Shanghai, China), and a $1 \mathrm{mM} \mathrm{HAuCl}_{4}$ solution was prepared in $0.2 \mathrm{M} \mathrm{Na}_{2} \mathrm{SO}_{4}$ for electrodeposition. All other reagents were of analytical grade and used without further purification unless otherwise stated. Deionized water $(\rho=18.2 \mathrm{M} \Omega \mathrm{cm})$ was obtained using a Pall (Port Washington, NY, USA) Cascada laboratory water system and was used in all the experiments. Environmental copper standard solutions (BY400031, BY40033) supplied by the Tanmo Quality Inspection Technology Corporation (Beijing, China) and calibrated by inductively coupled plasma optical emission spectrometry (ICP-OES) were used to validate and 
evaluate the new analytical method. The Chinese seawater standard GBW(E) 130011 with a salinity of 34.99 was purchased from the National Standard Material Network. Polyethylene bottles (Xiangyun Plastic Products Factory, Hebei, China) that were used to hold water samples were filled with $0.1 \mathrm{M} \mathrm{HCl}$ (analytical grade) for at least 1 day and then thoroughly washed with deionized water before use. Cellulose acetate membranes that were used to filter water samples were supplied by Shanghai Xingya Purification Material Factory (Shanghai, China).

\subsection{Sample collection}

Seawater samples were all collected from the same location, a coastal point in the Huanghai Sea (121.47 E, 37.49 N), which is significantly impacted by tidal and anthropogenic activity. Surface seawater samples (from depths of between 0 and $1 \mathrm{~m}$ ) were collected using pretreated $2.5 \mathrm{~L}$ sample collectors twice per day (at 08:00 a.m. and 14:00 p.m.). Once collected, the samples were immediately analyzed for temperature $(T)$ and $\mathrm{pH}$ and then transferred into individual $500 \mathrm{~mL}$ acid-cleaned polyethylene bottles.

\subsection{Sample pretreatment and determining the $\mathrm{Cu}$ speciation}

Water samples were immediately processed following collection according to the methodology summarized in Fig. 1. Water sample pretreatment was divided into three steps: filtration, acidification, and UV digestion. During filtration, the water samples were filtered through $0.45 \mu \mathrm{m}$ cellulose acetate membranes. Acidification involved the addition of $1 \mathrm{~mL} \mathrm{HCl}$ per $100 \mathrm{~mL}$ water in order to drop the $\mathrm{pH}$ value of the sample below 2.0. The sample was then maintained at $4{ }^{\circ} \mathrm{C}$ for $24 \mathrm{~h}$. An acidification period of $24 \mathrm{~h}$ to ensure that all of the acid-dissolved $\mathrm{Cu}$ had reached equilibrium was chosen based on the results of a previous experiment (Laglera et al. 2013). Acidification was performed to break down complexes of $\mathrm{Cu}$ with organic matter, releasing the $\mathrm{Cu}$.
The procedure also ensured that there was no metal adsorbed on the container walls during storage (Mart 1979; Patterson et al. 1976). In the UV digestion step, the acidified samples were transferred to quartz tubes and digested for $45 \mathrm{~min}$ under irradiation from a high-pressure mercury vapor lamp, which destroys all complexes and compounds and thus allows the total dissolved $\mathrm{Cu}$ concentration to be determined (Capodaglio et al. 1994, 1998). Samples that only underwent filtration as pretreatment were then immediately analyzed by anodic stripping voltammetry to avoid metal adsorption; direct electrochemical analysis was employed without $\mathrm{pH}$ adjustment to establish the concentrations of electroactive $\mathrm{Cu}$ species. Samples pretreated with filtration and acidification steps were analyzed by the same electrochemical method described above, but the overall concentration of acid-dissolved $\mathrm{Cu}$ and electroactive $\mathrm{Cu}$ in each marine sample was obtained. The concentration of acid-dissolved $\mathrm{Cu}$ was then derived by subtracting the previously obtained concentration of electroactive $\mathrm{Cu}$ species from the overall concentration of acid-dissolved $\mathrm{Cu}$ and electroactive $\mathrm{Cu}$. Finally, the total dissolved $\mathrm{Cu}$ concentration was determined for samples pretreated with filtration, acidification, and UV digestion. The concentration of inert $\mathrm{Cu}$ was then derived by subtracting the acid-dissolved $\mathrm{Cu}$ and electroactive $\mathrm{Cu}$ concentrations from the total dissolved $\mathrm{Cu}$ concentration.

\subsection{Electrochemical analysis}

Following the preprocessing steps, the samples were analyzed by electrochemical anodic stripping voltammetry using the electrochemical sensor. Before using it, a bare glassy carbon electrode (GCE) was polished sequentially with a 0.3 and $0.05 \mu \mathrm{m}$ alumina slurry, washed thoroughly with deionized water, and then ultrasonicated for $1 \mathrm{~min}$. The GCE was then subjected to potential cycling $(-0.2$ to $0.8 \mathrm{~V}$, $10 \mathrm{mV} \mathrm{s}^{-1}$ ) in $0.5 \mathrm{~mol} \mathrm{~L}^{-1} \mathrm{H}_{2} \mathrm{SO}_{4}$ until stable and reproducible cyclic voltammograms were obtained. Finally, the GCE was immersed in a $0.2 \mathrm{~mol} \mathrm{~L}^{-1} \mathrm{Na}_{2} \mathrm{SO}_{4}$ solution containing $1 \mathrm{mmol} \mathrm{L}^{-1} \mathrm{HAuCl}_{4}$ to electrodeposit a Au film onto the

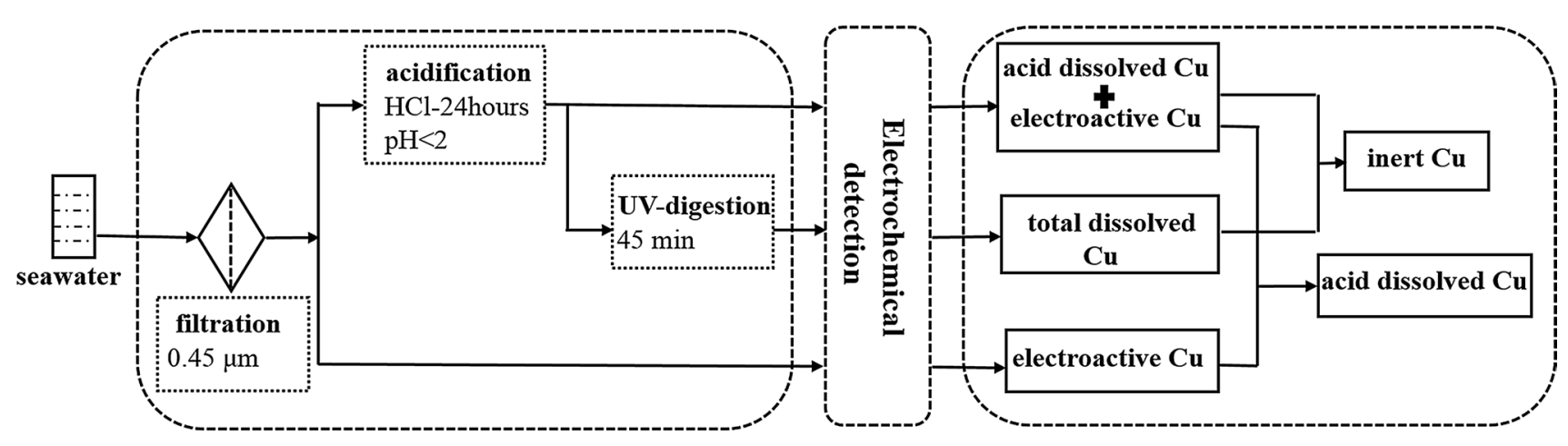

Fig. 1 Diagram showing the process used to analyze the speciation of $\mathrm{Cu}$ 
surface of the GCE, thus generating the working Au/GCE. The deposition parameters used were as follows: constant potential, $-0.2 \mathrm{~V}$; deposition time, $60 \mathrm{~s}$.

$\mathrm{Cu}$ speciation analysis was performed directly without further $\mathrm{pH}$ adjustment in $10 \mathrm{~mL}$ pretreated marine samples by anodic stripping voltammetry using the electrochemical sensor. This involved two main steps: accumulation and stripping out. First, $\mathrm{Cu}^{2+}$ was reduced to $\mathrm{Cu}$ on the surface of the $\mathrm{Au} / \mathrm{GCE}$ under a potential of $-0.4 \mathrm{~V}$ for $120 \mathrm{~s}$ with continual agitation. After an equilibration period of $10 \mathrm{~s}$, square-wave stripping voltammetry (SWV) was performed from 0.2 to $0.7 \mathrm{~V}$ in increments of $0.004 \mathrm{~V}$. It was found that the Au/GCE could be reused multiple times as long as it was electrolytically cleaned at a constant potential of $0.5 \mathrm{~V}$ for $10 \mathrm{~s}$ with slow stirring after each use. The concentrations of different $\mathrm{Cu}$ species were determined using the standard addition method.

\section{Results and discussion}

\subsection{Speciation of $\mathrm{Cu}$ in seawater}

Different $\mathrm{Cu}$ species were detected in the seawater samples, and relevant SWV current densities are presented in Fig. 2. $\mathrm{Cu}$ species concentrations obtained via the standard addition method are reported in the Electronic supplementary material (ESM). In Fig. 2, curve (a) was obtained for a sample pretreated with filtration only, so it shows the oxidation peak of electroactive $\mathrm{Cu}$. A large amount of organic matter was present in the sample, and this matter interfered with the electrochemical detection and distorted the shape of the peak. Conversely, the shapes of the acid-dissolved $\mathrm{Cu}$ curve (b) and the total dissolved $\mathrm{Cu}$ curve (c) are less distorted because additional acidification or digestion pretreatment steps were applied to the relevant samples. In addition to

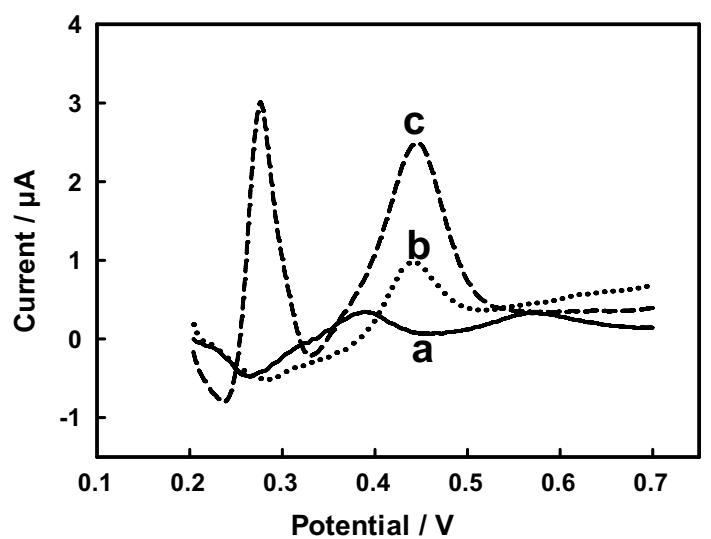

Fig. 2a-c Voltammetric curves of electroactive $\mathrm{Cu}$ (a), acid-dissolved $\mathrm{Cu}(\mathbf{b})$, and total dissolved $\mathrm{Cu}(\mathbf{c})$ in real seawater the peak from $\mathrm{Cu}$, another peak can be seen in curve (c). This can be attributed to the oxide species (bromine and chlorine) that are present in the seawater after UV irradiation. Acidification or UV irradiation destroys organic matter, releasing bound metal and ensuring that any adverse effects from the presence of organic matter on the voltammetric determination are eliminated (Mart et al. 1980; Annibaldi et al. 2011). The peak potentials of different species of $\mathrm{Cu}$ varied to a minor extent depending on the pretreatment method(s) applied. This can be attributed to the influence of the organic matter present in the samples on the rate of $\mathrm{Cu}$ oxide dissolution. It should be noted that the peak currents of the three $\mathrm{Cu}$ speciation groups differed significantly due to the varying $\mathrm{Cu}$ concentrations in the samples. The peak currents of different $\mathrm{Cu}$ species are closely related to both the content of organic matter and the physicochemical parameters of the natural water sample; $\mathrm{Cu}$ ions may react differently in different aquatic environments (Palma et al. 2016; van den Berg, 1982).

\subsection{Validation of the analytical method}

The method created in this work was used to analyze real seawater directly. A good stripping peak for $\mathrm{Cu}$ was obtained, demonstrating that this method is not influenced by the salinity of real seawater. Since this particular experiment was performed in seawater directly, it was impossible to explore the blank level of this method. In order to investigate the detection limit of this method, experiments were carried out with a seawater standard, and the detection limits of the method for electroactive $\mathrm{Cu}$, acid-dissolved $\mathrm{Cu}$, and total dissolved $\mathrm{Cu}$ were found to be $1.1,0.8$, and $0.5 \mathrm{nM}$, respectively.

To further validate the proposed analytical procedure, the method was applied to determine the total dissolved $\mathrm{Cu}$ in analytical standard reference materials and real environmental seawater samples. The concentrations of $\mathrm{Cu}$ in the environmental seawater samples determined using the electrochemical method were compared to the corresponding results obtained using ICP-MS (Table 1), and good agreement was observed. Before the ICP-MS determination, $0.5 \mathrm{~mL}$ of $10 \mathrm{mg} \mathrm{mL}^{-1} \mathrm{Ga}^{3+}$ were added to each $10 \mathrm{~mL}$

Table 1 Analytical results $(n=3)$ for the determination of $\mathrm{Cu}(\mathrm{nM})$ in certified environmental standard reference materials and an environmental sample

\begin{tabular}{lrll}
\hline Sample & \multicolumn{2}{l}{ Concentration of $\mathrm{Cu}(\mathrm{nM})$} \\
\cline { 2 - 4 } & Determined & Certified & ICP-MS \\
\hline BY400031 & $240.63 \pm 3.12$ & 235.43 & - \\
BY400033 & $97.93 \pm 2.67$ & 94.17 & - \\
Real seawater sample & $71.38 \pm 2.91$ & - & $66.33 \pm 0.78$ \\
\hline
\end{tabular}


seawater sample. Then, by adjusting the $\mathrm{pH}$ of the solution to 9.0 with ammonia hydroxide, interference from the salt in the water was eliminated through coprecipitation. After that, the solution was examined by ICP-MS using argon as a carrier gas, and the $\mathrm{Cu}$ in the solution was quantified by a standardized curve method. The measured concentrations of total dissolved $\mathrm{Cu}$ in the environmental standard reference materials were found to be in good agreement with the certified values within experimental error, showing that the measurements were accurate.

These findings demonstrate the applicability of this method to the direct determination of the total dissolved $\mathrm{Cu}$ in real seawater samples. The currently favored analytical method, ICP-MS, detects and quantifies the total dissolved $\mathrm{Cu}$ concentration against standard reference materials, and therefore does not quantify electroactive $\mathrm{Cu}$ or acid-dissolved $\mathrm{Cu}$. Since the same method was used to separately determine the electroactive $\mathrm{Cu}$, the acid-dissolved $\mathrm{Cu}$, and the total dissolved $\mathrm{Cu}$, and this method was validated, we can infer that the electroactive $\mathrm{Cu}$ and acid-dissolved $\mathrm{Cu}$ concentrations determined using the method were accurate.

\subsection{Reproducibility and repeatability}

If it is to be used for environmental monitoring applications, the sensor must be stable. Therefore, the reproducibility of the signal from the electrochemical sensor was checked by performing $\mathrm{Cu}$ detection in environmental seawater samples twenty consecutive times. Real seawater that contained $200 \mathrm{nM} \mathrm{Cu}$ was used, and the acid-dissolved $\mathrm{Cu}$ was determined as described above. After each determination, the Au/GCE was electrolytically cleaned and renewed by oxidizing any $\mathrm{Cu}$ that remained on the electrode. The observed fluctuation in the response of the

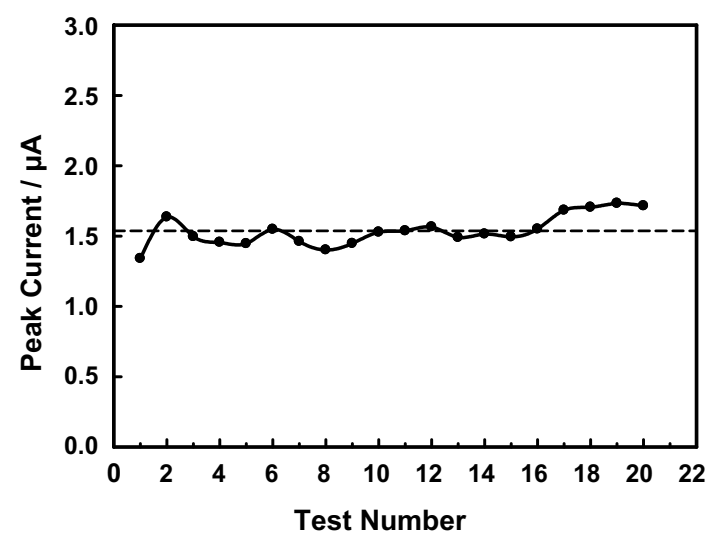

Fig. 3 Variance and reproducibility of the peak current from the electrochemical sensor upon continuous repeated use in real seawater containing $200 \mathrm{nM} \mathrm{Cu}$ ion. Dashed line is the average of all peak current values sensor across the twenty consecutive analyses is shown in Fig. 3. The horizontal dashed line in Fig. 3 is the average of the twenty determinations. The relative standard deviation (RSD) of the peak current was $7.05 \%$. This plot illustrates that the electrochemical sensor can be used for continuous analysis, as it shows high stability and reproducibility while detecting various $\mathrm{Cu}$ speciation groups.

The repeatability of the electrochemical sensor was investigated by determining the total dissolved $\mathrm{Cu}$ in real seawater samples containing $200 \mathrm{nM} \mathrm{Cu}$ each day across a seven-day period. The electrode was used twice each day (once in the morning and once in the afternoon). After each determination, the electrode was renewed through potential (electrolytic) cleaning and then immersed in deionized water until the next determination. All of the observed current signals are shown in Fig. 4, where the dashed line is the average of all the current values. The current produced by the electrode fluctuated more dramatically across the seven days than in the reproducibility experiment. This is because all of the measurements were performed in the same environment in the reproducibility experiment, so the variation in the current signal observed in that experiment is an almost direct reflection of the stability and repeatability of the electrode. However, the current fluctuations observed during the repeatability experiment were caused by both electrode instability and variations in the analytical matrix or experimental environment over the seven-day period. The RSD of the peak current during the seven days was calculated to be $11.71 \%$; further optimization should allow the sensor to meet the stability requirements for effective continuous monitoring.

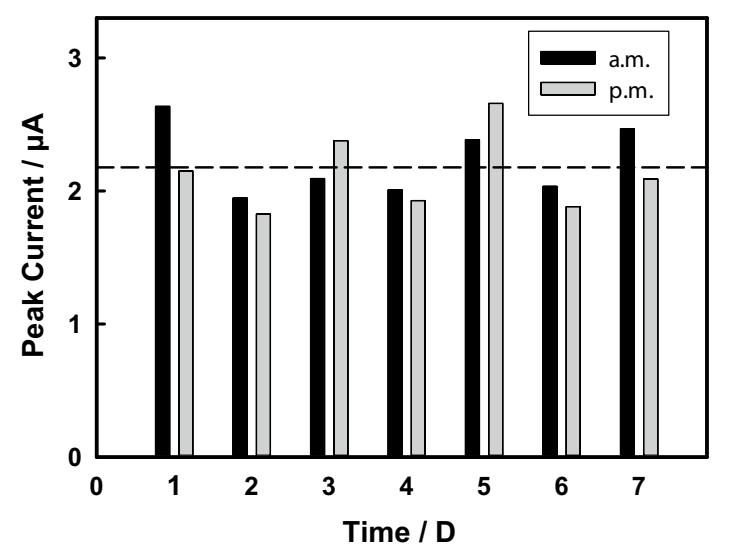

Fig. 4 Variance in and reproducibility of the peak current of the electrochemical sensor upon its continuous use in real seawater containing $200 \mathrm{nM} \mathrm{Cu}$ ion over a seven-day period. Dashed line is the average of all peak current values 


\subsection{Application to the determination of different $\mathrm{Cu}$ species}

Electrochemical stripping voltammetry was used to continuously monitor different $\mathrm{Cu}$ species in environmental seawater samples. Monitoring lasted for a 15-day period (1st March 2017 to 15th March 2017). Samples were collected at 08:00 a.m. and 14:00 p.m. each day, and the mean of at least three determinations was used for analyses. Relevant seawater characteristics and parameters as well as the concentrations and relative abundances of different $\mathrm{Cu}$ species are shown in Table S1 of the ESM, while a comprehensive summary of the concentration fluctuations is provided in Fig. 5. During days 1-5, the electroactive $\mathrm{Cu}$ concentration ranged from 5.91 to $14.87 \mathrm{nM}$, with an average concentration of $9.96 \mathrm{nM}$. The acid-dissolved $\mathrm{Cu}$ concentration ranged from 27.56 to $48.80 \mathrm{nM}$, with an average concentration of $36.45 \mathrm{nM}$, while the total dissolved $\mathrm{Cu}$ concentration

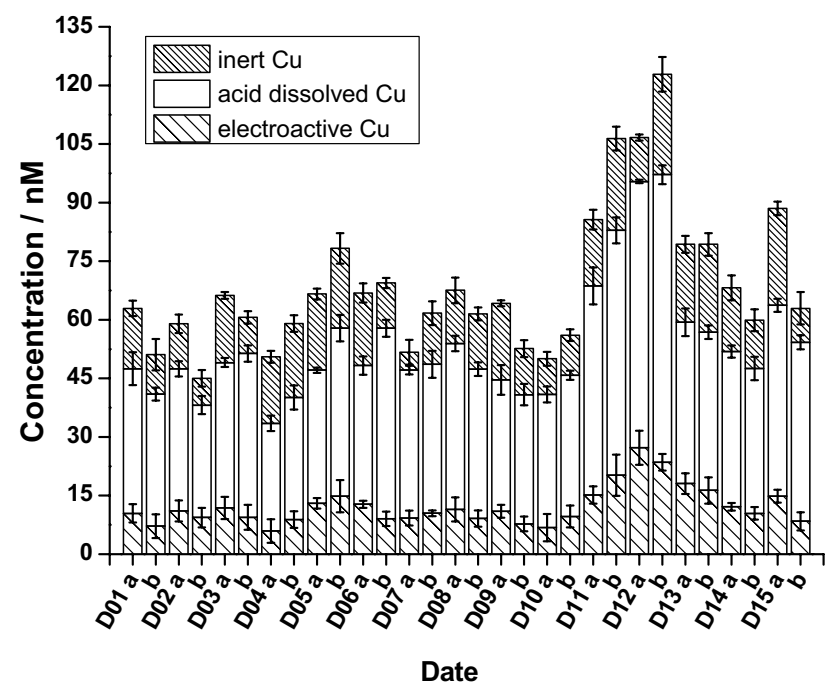

Fig. 5 Concentrations of different species of $\mathrm{Cu}$ detected in real seawater samples during the experimental period. Along the abscissa, bar "a" represents the morning and bar "b" represents the afternoon ranged from 45.03 to $78.28 \mathrm{nM}$, with an average concentration of $60.05 \mathrm{nM}$. There was no substantial change in the concentration of each $\mathrm{Cu}$ species between the first and second sampling event, although the largest $\mathrm{Cu}$ concentrations were measured on day 11 and over the following few days. This phenomenon may be ascribed to strong rainfall in the Yantai area on day 11, resulting in river runoff. This runoff washed $\mathrm{Cu}$ and dissolved organic materials into the seawater, causing significant increases in the concentrations of electroactive $\mathrm{Cu}$, acid-dissolved $\mathrm{Cu}$, and total dissolved $\mathrm{Cu}$ species. Also, the disturbance of the sediment by the rainwater would have led to the resuspension of organic matter, causing major fluctuations in the levels of different $\mathrm{Cu}$ species. The maximum concentrations of electroactive $\mathrm{Cu}$, acid-dissolved $\mathrm{Cu}$, and total dissolved $\mathrm{Cu}$ were observed to be $27.23,73.62$, and $122.85 \mathrm{nM}$, respectively. However, there was no obvious increase in the concentration of inert $\mathrm{Cu}$. As reported previously (Plavšić et al. 2008), the dissolved organic ligands present in rainwater during spring contain a particularly high number of active sites for $\mathrm{Cu}$ complexation, indicating that many of the organic ligands that complex with $\mathrm{Cu}$ in rainwater samples are not stable and can be destroyed by acid-based pretreatment methods. Three days after the rainfall, the concentrations of the different $\mathrm{Cu}$ species had generally returned to normal. Additionally, daily variations (i.e., fluctuations from a.m. to p.m.) in $\mathrm{Cu}$ concentrations were likely to have been due to tidal patterns, although specific patterns were unclear.

To further explore the correlations between the concentrations of different $\mathrm{Cu}$ species and the physicochemical parameters of seawater, a Pearson's correlation analysis (Wang et al. 2014) was performed, and the results are reported in Table 2. Significant positive relationships were found between the electroactive $\mathrm{Cu}$ concentration and the acid-dissolved $\mathrm{Cu}$ level $(r=0.83, p<0.01)$ as well as the total dissolved $\mathrm{Cu}$ level $(r=0.92, p<0.01)$. The correlations of the inert $\mathrm{Cu}$ concentration with the electroactive $\mathrm{Cu}$ level $(r=0.53, p<0.01)$, the acid-dissolved $\mathrm{Cu}$ level $(r=0.35$, $p<0.05)$, and the total dissolved $\mathrm{Cu}$ level $(r=0.65, p<0.01)$
Table 2 Pearson's correlation matrix for the concentrations of different $\mathrm{Cu}$ species and the physical and chemical parameters of seawater samples

\begin{tabular}{|c|c|c|c|c|c|c|}
\hline & $T$ & $\mathrm{pH}$ & Electroactive $\mathrm{Cu}$ & Acid-dissolved $\mathrm{Cu}$ & Inert $\mathrm{Cu}$ & $\begin{array}{l}\text { Total } \\
\text { dissolved } \\
\mathrm{Cu}\end{array}$ \\
\hline$T$ & 1.00 & & & & & \\
\hline $\mathrm{pH}$ & 0.35 & 1.00 & & & & \\
\hline Electroactive $\mathrm{Cu}$ & 0.07 & $0.34 *$ & 1.00 & & & \\
\hline Acid-dissolved $\mathrm{Cu}$ & 0.26 & $0.32 *$ & $0.83 * *$ & 1.00 & & \\
\hline Inert $\mathrm{Cu}$ & 0.09 & $0.37 *$ & $0.53 * *$ & 0.35 & 1.00 & \\
\hline Total dissolved $\mathrm{Cu}$ & 0.20 & $0.39 *$ & $0.92 * *$ & $0.93 * *$ & $0.65 * *$ & 1.00 \\
\hline
\end{tabular}

*Significant at the 0.05 level

**Significant at the 0.01 level 
were relatively poor. This may be because there was conversion of electroactive $\mathrm{Cu}$ into acid-dissolved $\mathrm{Cu}$ and vice versa under certain conditions, and both of these forms of $\mathrm{Cu}$ were directly related to the total dissolved $\mathrm{Cu}$. However, the inert $\mathrm{Cu}$ was very stable. Seawater is a typical multicomponent system that contains a large number of metal, inorganic, and organic ligands. There would have been competitive reactions involving the different species of $\mathrm{Cu}$ in seawater, which would have eventually reached equilibrium. Inert $\mathrm{Cu}$ was strongly bound to organic complexes, so the concentration of inert $\mathrm{Cu}$ would have been mainly determined by the types of inorganic and organic ligands present in the seawater. Positive relationships were also observed between the $\mathrm{pH}$ and the various $\mathrm{Cu}$ species concentrations because the correlation coefficients $(r)$ were all greater than $0.3(p<0.05)$. There were no significant correlations between $T$ and the concentrations of the various $\mathrm{Cu}$ species.

The relative abundances of the different $\mathrm{Cu}$ species comprising the total dissolved $\mathrm{Cu}$ were investigated. In complex environmental matrices, the abundances of different $\mathrm{Cu}$ species may be significantly driven by the types and concentrations of the organic species and matter present (Plavšić 2003; Schroeder et al. 2017). Electroactive $\mathrm{Cu}$ includes partially free $\mathrm{Cu}$ ions, which are believed to be the strongest influence on the bioavailability and toxicity of $\mathrm{Cu}$ (Guo et al. 2010; Brown and Markich 2000). Dissolved inorganic and organic ligands are abundant in aquatic environments. $\mathrm{Cu}$ exists mostly in inorganic and organic complexes, which decreases the fraction of $\mathrm{Cu}$ available to interact with organisms and to cause toxicity (Tait et al. 2016). Upon considering the type of dissolved organic material present (Plavšić et al. 2006), if the dissolved organic material forms stable complexes with $\mathrm{Cu}$ and those complexes are difficult to destroy, the percentage of inert $\mathrm{Cu}$ increases and the $\mathrm{Cu}$ becomes less ecotoxic. Additionally, the $\mathrm{Cu}$ in complexes destroyed by acid digestion shows low activity but can be transformed and absorbed by living organisms in certain cases, leading to a high risk of trophic transfer. Based on the findings shown in Figs. 5 and 6 , the dominant form of $\mathrm{Cu}$ in this region was acid-dissolved $\mathrm{Cu}$, which accounted for around $60 \%$ of the total dissolved $\mathrm{Cu}$, while the relative abundances of electroactive $\mathrm{Cu}$ and inert $\mathrm{Cu}$ were smaller (about 20\% each). This implies that, in the studied area, the $\mathrm{Cu}$ generally formed weak complexes with dissolved organic materials, and acidification destroyed most of the dissolved organic material, thus releasing $\mathrm{Cu}$. The variations observed in the levels of the $\mathrm{Cu}$ speciation groups (especially inert $\mathrm{Cu}$ ) were not substantial, which may be because all of the samples were collected from the same sampling location, thereby limiting potential variations due to changes in water quality conditions or organic matter content. The electroactive $\mathrm{Cu}$ and the acid-dissolved $\mathrm{Cu}$ levels fluctuated more sharply than that of the inert $\mathrm{Cu}$, further supporting the idea that the electroactive $\mathrm{Cu}$ and the

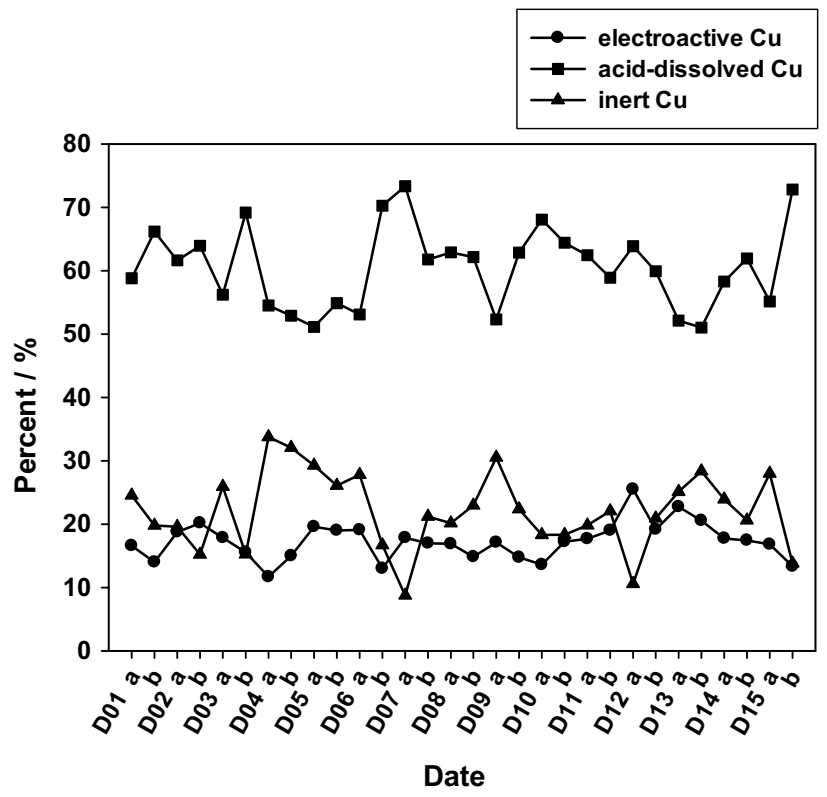

Fig. 6 Variations in the relative abundances of the different $\mathrm{Cu}$ species during the experimental period. Along the abscissa, bar "a" represents the morning and bar " $b$ " represents the afternoon

acid-dissolved $\mathrm{Cu}$ levels were more unstable and more readily influenced by the external environment than the level of inert $\mathrm{Cu}$. It is worth noting that while heavy rainfall was found to influence the total dissolved $\mathrm{Cu}$ concentration, no significant change in the ratio of the abundances of the $\mathrm{Cu}$ speciation groups was observed.

\section{Conclusions}

Electroanalytical techniques have been found to be useful and convenient for rapid metal speciation analysis. The present study effectively used a stripping voltammetric method to determine different $\mathrm{Cu}$ species during the direct analysis of seawater samples using an electrochemical sensor. The speciation analysis performed for this method does not require a complicated pretreatment process or separation operation, and the stability and reliability of this method have been effectively verified. Critically, the study has shown that this method can be used for the accurate longterm determination of various $\mathrm{Cu}$ species in complex seawater matrices, as the salinity of the matrix did not lead to any interference.

Based on the fixed-point monitoring results, it was concluded that without any changes in the environmental conditions, the relative abundances of the different $\mathrm{Cu}$ species would remain constant, and that the ratio of abundances was directly correlated with seawater quality and humus content. Rainfall influenced the ratio of the abundances of different 
$\mathrm{Cu}$ species because of the external input and the resuspension of sediment. Positive relationships were found between $\mathrm{pH}$ and the different $\mathrm{Cu}$ species concentrations, and no significant correlations were found between $T$ and the levels of the different $\mathrm{Cu}$ species. The strengths of the correlations between different $\mathrm{Cu}$ species varied greatly, although the concentration of electroactive $\mathrm{Cu}$ was found to be strongly positively correlated with the levels of other $\mathrm{Cu}$ species. The relationships of these concentrations to environmental and seasonal variations require further study.

Considering the importance of effective speciation analysis in natural water monitoring, this morphological classification and analysis method shows great promise, as it could provide a better understanding of the role of metal speciation in the marine environment.

Acknowledgements This research was supported by the Youth Innovation Promotion Association (2011170) and the Equipment Development Project (YZ201558) of the Chinese Academy of Sciences. We thank MogoEdit for its linguistic assistance during the preparation of this manuscript.

\section{References}

Abdelraheem WHM, Zanaty RK, Ismail NM (2017) Electrochemical determination of $\mathrm{Cu}^{2+}$ complexation in the extract of E. crassipes by anodic stripping voltammetry. Arab J Chem 10:S1105-S1110

Adnívia SCM, Corinne P, André HR, José PP (2016) Towards field trace metal speciation using electroanalytical techniques and tangential ultrafiltration. Talanta 152:112-118

Annibaldi A, Illuminati S, Truzzi C, Scarponi G (2011) SWASV speciation of $\mathrm{Cd}, \mathrm{Pb}$ and $\mathrm{Cu}$ for the determination of seawater contamination in the area of the Nicole shipwreck (Ancona coast, Central Adriatic Sea). Mar Pollut Bull 62:2813-2821

Batley GE, Florence TM (1971) A novel scheme for the classification of heavy metal species in natural waters. Anal Lett 9:379-388

Brown PL, Markich SJ (2000) Evaluation of the free ion activity model of metal-organism interaction: extension of the conceptual model. Aquat Toxicol 51:177-194

Byrne RH, Miller WL (1985) Copper(II) carbonate complexation in seawater. Geochim Cosmochim Acta 49(8):1837-1844

Capodaglio G, Toscano G, Scarponi G, Cescon P (1994) Copper complexation in the surface seawater of Terra Nova Bay (Antarctica). Int J Environ Anal Chem 55:129-148

Capodaglio G, Turetta C, Toscano G, Gambaro A, Scarponi G, Cescon P (1998) Cadmium, lead and copper complexation in Antarctic coastal seawater. Evolution during the austral summer. Int J Environ Anal Chem 71:195-226

de Polo A, Scrimshaw MD (2012) Challenges for the development of a biotic ligand model predicting copper toxicity in estuaries and seas. Toxicol Chem 31(2):230-238

Florence TM (1986) Electrochemical approaches to trace element speciation in waters: a review. Analyst 111:489-505

Gledhill M, van den Berg CMG (1995) Measurement of the redox speciation of iron in seawater by catalytic cathodic stripping voltammetry. Mar Chem 50:51-61

Grosell M, Wood CM (2002) Copper uptake across rainbow trout gills: mechanism of apical entry. J Exp Biol 205(8):1179-1188

Guo X, Ma Y, Wang X, Chen S (2010) Re-evaluating the effects of organic ligands on copper toxicity to barley root elongation in culture solution. Chem Speciat Bioavailab 22(1):51-59
Hirose K (1994) Conditional stability constants of metal complexes of organic ligands in sea water: past and present, and a simple coordination chemistry model. Anal Chim Acta 284:621-634

Hirose K (2006) Chemical speciation of trace metals in seawater: a review. Anal Sci 22:1055-1063

Huang S, Wang Z (2003) Application of anodic stripping voltammetry to predict the bioavailable/toxic concentration of $\mathrm{Cu}$ in natural water. Appl Geochem 18:1215-1223

Jacquot JE, Horak REA, Amin SA, Devol AH, Ingalls AE, Armbrust EV, Stahl DA, Moffett JW (2014) Assessment of the potential for copper limitation of ammonia oxidation by Archaea in a dynamic estuary. Mar Chem 162(20):37-49

Kot A, Namiesnik J (2000) The role of speciation in analytical chemistry. Trend Anal Chem 19:69-79

Laglera LM, Santos-Echeandía J, Caprara S, Monticelli D (2013) Quantification of iron in seawater at the low picomolar range based on optimization of bromate/ammonia/dihydroxynaphtalene system by catalytic adsorptive cathodic stripping voltammetry. Anal Chem 85:2486-2492

Mart L (1979) Prevention of contamination and other accuracy risks in voltammetric trace metal analysis of natural waters. Pt I: Preparatory steps, filtration and storage of water samples. Fresenius Z Anal Chem 296:350-357

Mart L, Nürnberg HW, Valenta P (1980) Prevention of contamination and other accuracy risks in voltammetric trace metal analysis of natural waters. Pt III: Voltammetric ultratrace analysis with a multicell system designed for clean bench working. Fresenius Z Anal Chem 300:350-362

Palma O, Kristijan V, Marta P, Irena C, Ivan Š, Tatiana M (2016) Voltammetry as a tool for rough and rapid characterization of dissolved organic matter in the drainage water of hydroameliorated agricultural areas in Croatia. J Solid State Electron 20:3097-3105

Patterson C, Settle D, Glover B (1976) Analysis of lead in polluted coastal seawater. Mar Chem 4:305-319

Plavšić M (2003) Electroanalytical techniques applied for studying the interaction of organic matter and particles with metal ions in natural waters. Anal Lett 36:143-157

Plavšić M, Lee C, Ćosović B (2006) Copper complexing properties of melanoidins and marine humic material. Sci Total Environ 366:310-319

Plavšić M, Orlović-Leko P, Kozarac Z, Bura-Nakić E, Strmečki S, Ćosović B (2008) Complexation of copper ions in atmospheric precipitation in Croatia. Atmos Res 87:80-87

Santore RC, Di Toro DM, Paquin PR, Allen HE, Metey JS (2012) Biotic ligand model of the acute toxicity of metals. 2. Application to acute copper toxicity in freshwater fish and Daphnia. Environ Toxicol Chem 20(10):2397-2402

Schroeder H, Fabricius AL, Ecker D, Ternes TA, Duester L (2017) Metal(loid) speciation and size fractionation in sediment pore water depth profiles examined with a new meso profiling system. Chemosphere 179:185-193

Tait TN, Rabson LM, Diamond RL, Copper CA, McGeer JC, Smith DS (2016) Determination of cupric ion concentrations in marine waters: an improved procedure and comparison with other speciation methods. Environ Chem 13:140-148

van den Berg CMG (1982) Determination of copper complexation with natural organic ligands in seawater by equilibration with manganese dioxide. I: Theory. Mar Chem 11:307-322

Wang Z, Yao L, Liu G, Liu W (2014) Heavy metals in water, sediments and submerged macrophytes in ponds around the Dianchi Lake, China. Ecotoxicol Environ Saf 107:200-206

Whitby H, van den Berg CMG (2015) Evidence for copper-binding humic substances in seawater. Mar Chem 173:282-290 